\title{
The Electromagnetic Protection Method on the Guided Ammunition
}

\author{
Wengeng Pan, Hua Wan, Guangrong Bian and $\mathrm{Hu}$ Chao \\ Department of Aviation Ammunition, Air Force Logistics College, Jiangsu Xuzhou, China
}

\begin{abstract}
In response to the quality and the ability to survive for the guided ammunition in a complex electromagnetic environment, the guided ammunition quality for storage and use are analyzed from the design of the ammunition, structure wiring and protective material for the static electricity, lightning electromagnetic pulse and outside electromagnetic sources. It is proposed for the electromagnetic shielding method and protection countermeasures in order to ensure the electromagnetic safety of ammunition. It has military significance and economic value.
\end{abstract}

Keywords-ordnance depot; guided ammunition; electromagnetic protection; methods

\section{INTRODUCTION}

The ammunition was usually made up of electronic components and explosive system etc, it widely used in hightech ammunition for the integrated circuit as the core of microelectronics components and was of the characteristics for the micro power consumption, high density, high integration and high magnetic sensitivity. It was easy to damage or failure caused by the electromagnetic pulse disturbance and reduced the working life of the electronic equipment or lead to major accidents. Especially in high power radar and electric power equipment such as the presence of radiation source, the ammunition often threat such as nuclear electromagnetic pulse radar and electromagnetic pulse hazard sources as electric arc discharge. It was of great significance for the ammunitions especially guided ammunition electromagnetic protection issues to improve ammunition safety.

\section{Electrostatic Protection ON the Storage AMMUNITION}

Electrostatic discharge was a common hazard sources in near field electromagnetic pulse, microelectronics components of all kinds of ammunition was vulnerable to the interference of electromagnetic pulse resulted in damage so as to cause harm to stock ammunition. Stock ammunition was many static influenced such as the radio fuze of the ammunition. It should encounter all sorts of different static environment at the various stages of the radio fuze. When the radio fuze and warhead flying through the clouds, it may have strong electrostatic and damage the normal work of the radio fuze electronic components, initiating components from electrostatic interference and could also be advanced ignition. Minimum electrostatic energy of ammunition was one of the important data for the dangerous goods or job and explosion accident should happen. The main measures of electrostatic protection were the conditions for formation of electrostatic hazards and energy coupling way:

1) Controlling of electrostatic electrification rate could prevent the formation of dangerous static power. Electrostatic protection to prevent from the source, the key was to eliminate or reduce the generation of electrostatic charge and accumulation by reducing of producing electrostatic processes and materials in the work, it should not happen for the ammunition to keep the same potential or zero potential electrostatic discharge. A charged body was one of the static electricity danger in ammunition warehouse. To eliminate the human body static electricity was the important content for the anti-static measures in the ammunition storehouse. Ammunition warehouse was used anti-static cement plaster on the ground not to use insulating material such as rubber, plastic, asphalt paving, also did not allow the use of insulating coatings on the ground. Workers should wear the conductive shoes, antistatic shoes and not to wear the bottom of the mat or in plastic, rubber, insulation shoe with insoles. Not allowed to wear ordinary wool, silk, chemical fiber fabric garment. Special operations were not allowed to run and jump, change clothes, and pay attention to rhythm operation to avoid excessive separation of violent action.

2) It increased the charge dissipation rate for taking the humidifying, grounding and electric equipment or elimination measures to prevent charge accumulation and could eliminate electrostatic hazard when increasing the relative humidity in the workplaces. It could be dissipated or neutral when all the static charge couldn't eliminated in the work. It could effectively electrostatic discharge equipment, limit equipment build-up of electrostatic potential, also could prevent to produce spark discharge and static electricity charge by tools and makes a reliable electrical connection by the earth with the equipment that between the earth and its component of electrostatic charge leakage channels. Grounding could not eliminate electrostatic for some insulation materials and it was available for electrostatic ion function to counteract these materials.

3) Using antistatic discharge design technology improved the antistatic ability of the circuit and its equipment. It provided appropriate input to protect sensitive components in the process of the development of the components used and circuit as far as possible using electrostatic sensitive components or the use of electrostatic discharge. It eliminated electrostatic discharge damage to equipment and ammunition with antistatic discharge 
design, integrated use of grounding, lap, shielding, filtering, other hardware and software of electrostatic protective reinforcement technology too.

4) It all could heighten drug antistatic ability for the use of electrostatic insensitive such as initiating agent or adding the right amount of boron ignition powder containing an amorphous boron nitride and lead mixed medicine and joined the Sorbose and Cinnamic Acid ester $(0.001 \sim 0.005)$ in the nitride lead adding a small amount of Polyols. With hydrogenated titanium and potassium chlorate, combined antistatic ignition powder was a kind of hot resistant powder, resistant to $0.6 \mathrm{~F}, 25 \mathrm{KV}$ of electrostatic discharge, Three five vanadium oxide, alumina, aluminium coated potassium perchlorate mixing drugs, tolerated feet between the shell and $25 \mathrm{kv}$ multiple electrostatic discharge, Lead with graphite coated and phenolic acids, could reduce the accumulation of static charges, were the effective ways to reduce electrostatic hazards.

5) It was an important part of the warehouse safety for strengthening the static safety management. Ammunition warehouse, ammunition repair station and waste processing center should formulate relevant rules and regulation and establish the technical measures of anti-static hazard and safety system. For static electricity grounding device should be regularly check maintenance to prevent its chemical corrosion and mechanical damage. It could meet the requirements for repair or replacement in time by measuring grounding resistance and leakage resistance regularly. Finally, to education of staff and improve anti-static consciousness [1,2].

\section{PRotection ON THE OUtSide ElectromagnetiC SOURCES}

General electromagnetic pulse energy was not easy to pass through the projectile into the internal due to electromagnetic waves penetrate through skin effect on metal shell. It should produce electromagnetic scattering near hole seam and the coupling phenomenon and play in PCB or components interact to produce electromagnetic pulse effect through a hole slot coupling into its internal only. Therefore, the ammunition for electromagnetic pulse protection should be considered in the design phase.

1) The round hole or a square hole was used as far as possible in fuze window, window and cable hole of the projectile design which helps to improve the shielding effectiveness of projectile. It could effectively reduce the influence of the external electromagnetic pulse of projectile and reduce the coupling into the play of electromagnetic energy in the body, it should adopt array hole when a porous seam must be opened.

2) The electromagnetic sensitive components should as far as possible away from the seam hole and cavity edge. It was prone to electromagnetic enhancement effect in seam hole and cavity near the edges. In layout components design of the body, electromagnetic sensitive components should be as far as possible to leave seam hole and cavity edges to avoid electromagnetic energy was high and the relatively dangerous place, it helps to improve the system's resistance to outside electromagnetic pulse.
3) Line cloth to should as far as possible in setting the body circuit and perpendicular to the axis of the projectile was parallel to the direction of the long sides of the rectangular hole to reduce the coupling field effects on lines or electronic components. Connect wires and wiring isolated sensitive components such as the power cord, line, high level and low level line should be isolation, and try to avoid parallel to each other. The effect of high frequency radiation that pieces of wiring and antenna effect also note especially. For through the magnetic field coupling of the radiation to increase the impedance of the circuit to reduce the interference coupling, the radiation should be reduced to electric field coupling circuit impedance coupling to reduce interference.

4) The circuit protection of electric ignition system was necessary in ammunition design. When suddenly a large energy of electromagnetic pulse, the protection circuit could be transient conduction and should have most capacity to destroy electromagnetic energy drains off so that the electric ignition of the electric ignition system protected and avoid to angry. Under the effect of RF, RF signal bridge circuit reactance balance precision balance to appear on both ends of the heating element such as potential, thus preventing radio frequency, static and thermal initiation problems caused by stray voltage. And also could use ignition head outside insulation coating conductive coatings, the spark was uniformly distributed throughout the ignition head of conductive layer on the outside in order to reduce electrostatic energy density. The characteristics of these devices was in the high voltage resistance was small, quick response and electromagnetic energy drain off instantly, and should not affect the normal ignition electric ignition system under the condition of low voltage state of high resistance. When the frequency of electromagnetic pulse and projectile coupling resonance frequency was same or similar, the electromagnetic pulse energy coupling was larger and the shielding coefficient of projectile in the body was lowest. It should as far as possible to avoid the resonance frequency of the cavity in design of circuit frequency response.

Shielding effectiveness of electromagnetic shielding was directly related to the characteristics of the material and the method of shielding of electromagnetic pulse were many, but most way could achieve shielding through the coating, coating, metal spray, vapor deposition and electroless plating[3,4]:

1) Shielding coating consists of synthetic resin, conductive fillers, solvents made from mixing. The spraying in play in the body, the outer surface, after dry could form the lacquer layer, to enhance the corrosion resistance and strength of the projectile, but also increased the electromagnetic performance. Adding different metal coating conductive filler, could produce different electrical conductivity of the coating. Generally use the metals such as gold, silver, copper, nickel powder or graphite powder. Gold, silver coating shielding was better, but the price was higher; Copper conductivity relatively was $10^{-2} \Omega \cdot \mathrm{cm}$, but copper in the coating was not stable, easy to loss of oxidation; Nickel coating was not easy oxidation. The main products in Europe and the United States against the electromagnetic shielding, the coating layer thickness was $50 \sim 70 \mathrm{um}$, conductivity resistivity was $10^{-3} \Omega \cdot \mathrm{cm}$, shielding effect could reach $30 \sim 60 \mathrm{~dB}$; Graphite coatings conductivity 
were relatively poor, shielding effect was poor. Also could join the conductive fillers such as silver plated copper powder surface, its conductivity relatively was $10^{-2} \Omega \cdot \mathrm{cm}$.

2) Flexible conductive medium mainly includes conductive rubber cushion (article), conductive cloth, wire mesh, etc. Conductive gasket could be implemented in aperture place electrical contact to achieve the continuity of the conductive was one of the effective ways to prevent crack electromagnetic leakage. Conductive film was the use of molten metal injection, the electroless plating coating and metal foil make the projectile get very thin layer of conductive metal surface and so on. Method of metal spray coating of metal layer thickness was $70 \mu \mathrm{m}$ and good electrical conductivity was $10^{-2} \Omega \cdot \mathrm{cm}$, electrical conductivity could be achieved for shielding effect $70 \mathrm{~d} B$. The electroless plating coating was copper, copper nickel alloy plating on plastic surface, coating thickness of about $50 \mu \mathrm{m}$, coating good electrical conductivity, strong bonding force, the shielding effect could reach $60 \mathrm{~dB}$. Stick a metal layer was the aluminum foil and copper foil on the plastic sheet etc. or film pressure into composite materials, shielding materials was suitable for soft and hard materials. In addition to the metal foil cover, it also could be used between wood or plastic, adhesive and good performance, good conductivity and its shielding effect could be as high as $60 \sim 70 \mathrm{~dB}$.

3) The material electromagnetic has protection ability to join the material such as the conductive glass fiber or carbon fiber conductive in ordinary material, to join the conductive glass fiber of $50 \% \sim 70 \%$, conductivity could be reached $10^{-2} \Omega \cdot \mathrm{cm}$, the shielding effect could reach (50 60) dB, but also greatly enhance the composite materials tensile strength, bending strength and heat resistance, in need of high light transmittance window for electromagnetic shielding, could choose glass (commonly known as shielding glass) of metal mesh compound visual shielding plate such as conductive glass Windows shielding material. Conducting packing could also be used in the ordinary materials of metal, carbon fiber, superconductive carbon black, metal alloy, polyaniline conductive fiber etc. Seams in sheet metal pieces of conductive rubber block sealing groove installation was adopted, the conductive rubber with conductive adhesive silicone adhesive on the metal components, could greatly improve the shielding effectiveness[5,6].

\section{Lightning Protection On the AMmunition STOCKPILES}

With the improvement of weapons and equipment level of electronic, ammunition and other weapons and equipment of lightning harm accident were growing. Once induced voltage, the induction current exceeds the components damage threshold, it could damage components, the interference of light make the system normal operation, the weight would cause the failure of weapons and equipment, it puts forward new requirements for the lightning protection of ordnance store $[7,8]$ :
1) Lightning electromagnetic pulse protection as an important part of the ordnance storehouse safety storage must full attention. Although in recent years, the aviation ordnance ammunition warehouse took some lightning protection measures such as installation of lightning rod, but there were serious lightning damage accidents. Simple for lightning electromagnetic pulse protection facilities, the advanced weapons should not be warehouse storage of ammunition with the management level couldn't keep up.

2) The active adoption of new technology of lightning protection could improve the effect of lightning protection. The effect of lightning rod was outstanding height make thunder clouds under the electric field distortion, should thunder upon them, and make protection from lightning strikes. We could use advanced lightning protection technology, the use of corona discharge way to neutralize thundercloud charge, weakened by lightning activity, reduce the number of lightning strike. For not fully charge, the thunder clouds could largely reduce ray current amplitude and steepness. This make the most of the energy consumption in the upper portion of the lightning rod, thus greatly reduced the current lead, so as to reduce or avoid the harm of thunder and lightning.

3) For the reservoir area of the lightning protection environment, it mainly consider three aspects of the address of the warehouse, warehouse building and the reservoir area such as green. Warehouse address should choose in less minefield, and try to choose good mountains area in natural conditions, under the slopes of soil should be has good electrical conductivity. Building form of Warehouse had the ground, underground structure and half underground repository. It should choose as far as possible half underground library from the perspective of lightning protection, the plains and desert area to store flammable and explosive goods warehouse. We should choose as far as possible underground structure in a mountainous area. In hilly region, we could choose underground structure, half underground library or the ground base. For half underground library near side, above the underground structure should be paid attention to and should not be set up and into all kinds of metal facilities should not be prominent planting of trees to avoid triggering lightning effect. Reservoir area of afforestation, tree species selection, planting density was closely related to the distance from the center of the warehouse and lightning protection, we must be taken into account. A variety of evergreen coniferous forest around the reservoir area could form a myriad of corona discharge tip, we make the thundercloud charge and land has numerous and channels. Highly prominent trees easily become the target of a lightning strike and causing a fire. So the trees around the storage and warehouse should be paid attention to keep a certain distance etc.

Lightning electromagnetic pulse protection was more difficult for the field ammunition supply, because field ammunition depot protection facilities was simple and its security was poorer compared with the rear ordnance store obviously. Especially in the south and south-east China thunderstorm period was longer and each year about $90 \sim 110$ days of thunderstorm. As a result, it should attach great focus on to the thunder and lightning electromagnetic pulse protection of field ammunition depot to prevent the occurrence 
of lightning accident. Basically has the following several aspects $[9,10]$ :

1) It must have the lightning protection facilities for the field ammunition depot aerial bombs in open air or use of temporary storage warehouse in order to avoid the lightning attacks and prevent thunder and lightning disasters. It usually adopts the lightning rod, wire and lightning arrester and so on. But it must be for lightning protection equipment regular check no matter adopt what kind of lightning protection device for protection to ensure that the grounding resistance shall not be exceeded $10 \Omega$.

2) Field ammunition depot should be a priority as far as possible use or had certain shielding effect of the building, home was best for the reinforced concrete structure to make the induced current smooth leak out and reduces the damage effect of lightning electromagnetic pulse. The cable were shielded cable should be used to block or wear iron pipe to prevent strong thunder and lightning electromagnetic pulse induction in ammunition signal lines and power transient overvoltage. When demand signal cable grounding in the end only, two layers of shielding should be adopted, the two end of outer shield connected to the electronic device shell respectively and nearby grounding, lightning electromagnetic impulse overvoltage could be greatly reduced.

3)Field ammunition depot should avoid inside separate houses, trees, antenna and water tower to thunder clouds with the plasma channel of tall or by independent buildings, the ammunition set to cylindrical as far as possible and make it has no obvious prominent sharp point. Due to lightning current was mainly along the resistivity of the smaller flow path, thus lightning location often occur in the soil resistivity smaller. When selecting configuration of field ammunition depot area in thunderstorm season, we should take the initiative to avoid the "lightning strike zone" in order to reduce the incidence of lightning strike accident.

\section{CONCLUSIONS AND RECOMMENDATIONS}

It study respectively the protection of the thunder, lightning and general electromagnetic pulse in the storage of ammunition and the outside ammunition and puts forward some protective measures of electromagnetic pulse according to the characteristics of static electricity and lightning electromagnetic pulse and general electromagnetic pulse from the design of the ammunition. We should choose reasonable holes seam types, electromagnetic sensitive components as far as possible away from the holes seam to optimize the design of the wiring and play the body design, protection circuit and the response frequency of the play within the circuit to avoid shielding cavity resonance frequency in the design of the ammunition. It is the most commonly used and most effective inhibition of electromagnetic pulse technology, but couldn't achieve the full screen, for some window and hole seam etc. in fact, we should be metallized film or glass of metal mesh screen, it is used for the in and out of the cable, wire and installed cable protector, filter, such as lap and grounding measures for all cables and pipelines in and out of the hole to be blocked with the electromagnetic wave absorption material. It could improve the electromagnetic compatibility ability of ammunition by natural electromagnetic compatibility design and shielding structure integrity and grasp the impact of electromagnetic pulse on ammunition security threshold for comprehensive analysis and research to the electromagnetic influence of the ammunition, all kinds of test result has to be an actual electromagnetic pulse test to ensure the security of the ammunition electromagnetic pulse protection.

\section{REFERENCES}

[1] Chen Rui-min. Antistatic-electricity Research on the explosive component[D]. Nanjing university of science and technology, 2008:2122.

[2] Liu Shang-he, Wu Zhan-cheng. Electrostatic discharge and hazard prevention[M]. Beijing university of posts and telecommunications publishing house, 2004.

[3] Gu Ying-ying,Chou Xiao-yong, Hu Qi-ming.The research progress of electromagnetic shielding material[J]. Material review,2005(2):53-56.

[4] Liu Tian-xin, GuanYing-xiang, Ma Hong-xuan. The research on the hardening technique for electrical initiating explosive device for a fuze to resist electromagnetic environmental hazards [J]. Journal of detection and control,2002(1):23-26.

[5] Li Jin-ming, An Zhen-tao, Luo Xing-bo. The effect of radio frequency to eed and its protection measures[J].Blasting equipment,2004(5):17-19.

[6] Yi Jian-zheng,Tan Chao-bing,Cai Jun-feng. Study of electromagnetic harm protection for rocket electrical ignition system[J]. Equipment environmental engineering,2006(4):52-54.

[7] Zhao Jian-yu,Tang Bao-hua. Lightning protection of rear ammunition warehouse[J].Warehouse management and technology,2003:78-79.

[8] Zhang Jin-sheng,Chen Ri-hai. Ordnance store lightning harm and its protection[J]. Warehouse management and technology,2003.

[9] Tian Yang-meng.The generating mechanism of lightning electromagnetic pulse, harm and its protection[J].Physics and engineering,2006(5):36-37.

[10] Ma Hui, Zhao Jun. The field ammunition lightning harm and protection $[\mathrm{J}]$. Equipped with environmental engineering,2006(2):80-81. 